\title{
DIFFICULT DIAGNOSIS IN A CASE OF TRANSVERSE COLON NEOPLASM INVADING THE GREATER GASTRIC CURVATURE
}

\author{
CIPRIAN TĂNĂSESCU ${ }^{1}$, MIHAI FAUR ${ }^{2}$, CLAUDIA GHERMAN ${ }^{3}$ \\ ${ }^{1,2}$ Sibiu County Clinical Emergency Hospital, ${ }^{3}$ “Iuliu Haţieganu” University of Medicine and Pharmacy Cluj Napoca
}

\begin{abstract}
Keywords: $\quad$ transverse Abstract: Colon cancer is considered to be the third most common neoplasm in men, while in women colon neoplasm, gastric it is considered the second most common cancer, according to the World Health Organization, the invasion, anemia, Globocan database. Colon adenocarcinoma represents over $90 \%$ of malignant tumours of the colon. parafluid mass, IC It is a malignant epithelial tumour originating in the epithelium of the colonic mucosa, which resection gradually invades all the layers of the colonic wall (the muscle of the mucosa, submucosa, own muscle, being able to surpass the serosa). We presented the case of an 80-year-old female patient with multiple comorbidities, admitted to a medical department, then transferred to the surgery department, whose definitive diagnosis was hardly made intraoperatively, despite multiple preoperative investigations performed in the two wards. The postoperative evolution was favourable. The case and the elements from the specialty literature are discussed, but also the particularities of the treatment of colonic cancer in the elderly and the possible complications arising postoperatively.
\end{abstract}

\section{INTRODUCTION}

Colon cancer is considered to be the third most common neoplasm in men, while in women it is considered the second most common cancer, according to the World Health Organization through the Globocan database. Globally, the incidence of colon cancer varies depending on dietary habits, exposure to environmental pollution, but also on the genetic risks or possible precancerous conditions in the personal medical history. $(1,2)$

The increased risk factors for colon cancer are: countries with low socio-economic development, sedentary, obese persons, unhealthy eating, smoking etc. $(3,4)$

Age is not a specific risk factor in colon cancer. It is rarely until the age of 40 . Afterwards, the incidence begins to increase significantly until the age of 50, subsequently increasing with each decade.

The survival of patients with colon cancer at 5 years from diagnosis is constantly increasing due to the early detection of the disease, but also due to the much more efficient treatment, both surgical and adjuvant. Thus, in the US, the 5-year survival rate in colon cancer, regardless of stage, reached $61 \% .(5,6)$

As a result of the increase in life expectancy, the age at which this condition appears has also increased. Most of the time, however, due to the existence of the associated age-related comorbidities, the treatment of elderly patients with colon cancer should be less aggressive but observing the oncologic principles as much as possible.

\section{CASE PRESENTATION}

We present the case of an 80-year-old female patient with multiple comorbidities: grade II hypertension, type 2 diabetes mellitus, stage II chronic kidney disease, gastric ulcer, cholecystectomy, ischemic heart disease, exercise-induced angina, aortic stenosis, cervical-dorsal-lumbar spondylosis, osteoporosis, who presented in the Emergency Room with stabbing pain in the chest in the left part, asthenia, fatigue, vertigo, expiratory dyspnea, symptomatology that started approximately one week ago.

Objective examination: altered general condition, underweight, afebrile, pale teguments and mucous membranes, saburral tongue, pain in the cervical-dorsal-lumbar spine, kyphoscoliosis and pain in the left hemithorax, expiratory dyspnea; decreased vesicular breath sounds, crepitant crackles, blood pressure $(\mathrm{BP})=140 / 80 \mathrm{mmHg}$, atrio-ventricular $(\mathrm{AV})=60 \mathrm{bpm}$; supple, mobile, sensitive to palpation abdomen in the epigastrium, intestinal transit present, unpalpable liver and spleen, free renal lodges, no Giordano sign, spontaneous urination. The patient was time and space-oriented.

At the palpation of the abdomen, in the left flank and iliac fossa, a thickening was felt, without sensitivity spontaneously and on palpation.

Biochemically, severe normocytic hypochromic anemia $(\mathrm{Hb}-7.3 \mathrm{~g} / \mathrm{dl})$ was noticed, as well as thrombocytosis $(543,000 \mathrm{ul})$, inflammatory biological syndrome, hydroelectrolytic disorders.

The patient was admitted to the Medical ward where the correction of anemia started by administrating erythrocyte and iron concentrate, diuretics, antibiotics, hydro-electrolytic rebalancing therapy, antiemetic, hepatic and gastric protection therapy, chronic cardiac therapy, under which the symptoms were slightly improved.

Due to the persistence of the symptoms, a pelvic abdominal CT was decided and performed that showed an elongated stomach with $4 / 8 \mathrm{~cm}$ parafluid mass that entered into close contact with the gastric wall on the large curvature, accumulation also described in the line joining the iliac crests, reason for which a surgical consultation, was requested after which, on 18.11.2019 the referral to the Surgery ward was

${ }^{2}$ Corresponding author: Mihai Faur, B-dul. C. Coposu, Nr. 2-4, Sibiu, România, E-mail: mishu.faur@yahoo.com, Phone: +40743805073 Article received on 27.03.2020 and accepted for publication on 28.05.2020 


\section{CLINICAL ASPECTS}

decided in order to establish the specialized surgical treatment.

Figure no. 1. Abdominal and pelvic CT

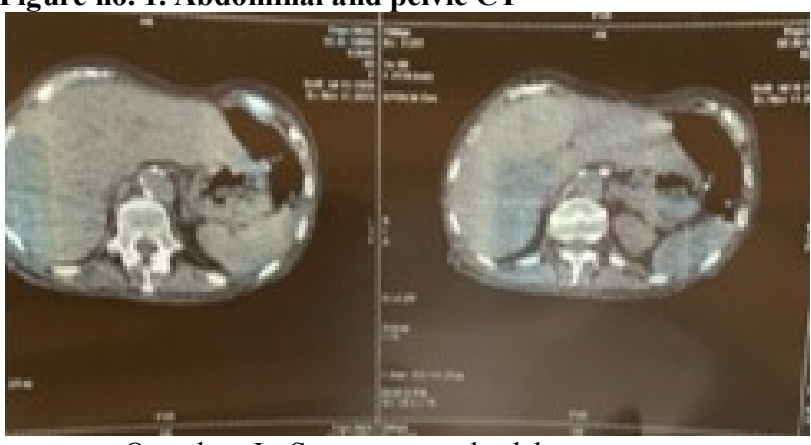

On the I Surgery ward, laboratory tests were performed that highlighted: Iron $210 \mathrm{ug} / \mathrm{dL}$; Leukocytes 10,850 ; Hemoglobin $10.6 \mathrm{~g} / \mathrm{dL}$; Thrombocytosis 568,000u/1.

Eco-endoscopy described: upper abdominal formation near the large gastric curvature with posterior wall of hypoecogenic consistency - suspicion of intraparietal gastric tumour.

The ultrasound showed a tumour formation located in the left flank and iliac fossa in close contact with the large gastric curvature.

After an adequate preoperative preparation, surgical intervention was performed in general anesthesia and endotracheal intubation, performing an exploratory laparotomy that showed a large tumour formation at the level of the transverse colon that invaded the large gastric curvature, multiple coloepiploic adhesions, for which segmental transverse colon resection was performed with IC resection of the stomach on the large curvature with a linear stapler (figures no. 2 and 3), with ascending-descending T-T anastomosis and closure of the mesenteric gap. Viscerolysis, washing, double drainage, parietoraphy in anatomical layers, sterile dressing were practiced.

Slow favourable postoperative evolution, patient with good general condition, stable in terms of cardio-respiratory status and hemodynamically, afebrile, supple, mobile abdomen, painless spontaneously and on palpation, post-operative healed wound, without reaction, permeable drainage tubes with minimal drainage, reason for which on the $6^{\text {th }}$ postoperative day they were suppressed, intestinal transit resumed for fecal matter and gas. postoperatively.

The patient was discharged on the $8^{\text {th }}$ day

\section{Figure no. 2. Colonic tumour}

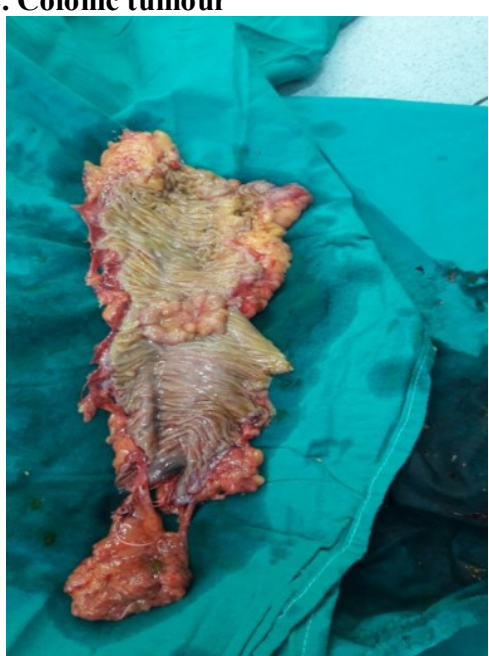

\section{Figure no. 3. Gastric invasion}

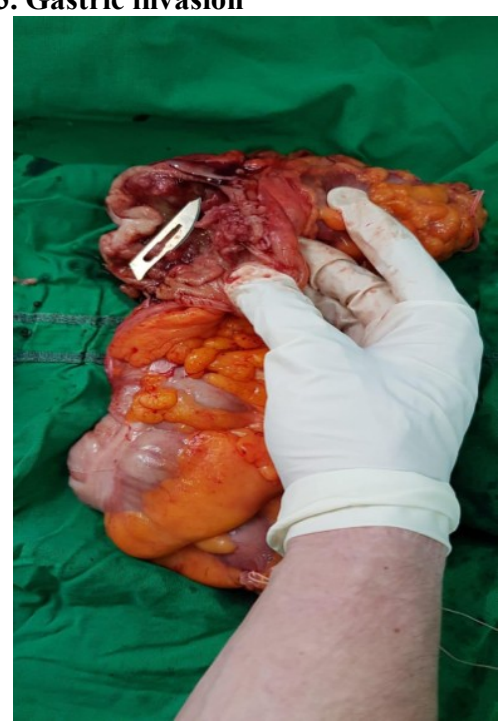

DISCUSSIONS

Colon cancer can be localized at different levels of the colon: caecum, ascending, transverse, descending, or sigmoid. It is an increasingly common neoplasm, both in men and women. Although the frequency of the disease is increasing, survival rate is improving, on the one hand due to the development of screening programmes that have led to the discovery of the disease in increasingly incipient stages with the possibility of establishing curative treatment, and on the other hand the development of complementary therapeutic methods: chemotherapy, radiotherapy, immunotherapy.

Unfortunately, in our country, both due to the lack of screening programmes for this pathology, as well as due to the patients' presentation to the doctor in advanced stages, the treatment is often curative, and the life expectancy is much lower.

Most frequently, colon cancer develops by malignant degeneration of adenomatous polyps. It is the stage in which the treatment of colon cancer can be curative. Unfortunately, even in the countries that have very well developed colon cancer screening programmes, the presentation to the doctor in the metastatic stage is up to $20 \%$.(7)

From the clinical point of view we have a differentiation of symptoms, thus in the right colon cancer the large tumour formations predominate, with secondary anemia, without intestinal occlusion, whereas in the left colon cancer the tumours are small, scirrhoid, the patient presenting most commonly in the emergency department with intestinal occlusion or subocclusion.

Unfortunately, in the case of tumours of the transverse colon the symptomatology is quite poor, the patient presenting to the doctor quite frequently in the metastatic stage, complaining about the increase in size of the abdomen based on the tumour size or the presence of ascites, feeling of early satiety, cutaneous metastases or signs of malignant impregnation.

Colon cancer can be spread in 4 directions: lymphatic metastasis in locoregional lymph nodes, hematogenous dissemination in liver and lungs, bones, intraperitoneal dissemination and dissemination by loco-regional invasion.(7) In our case, metastasizing was extremely rapid through locoregional invasion at stomach level, and the symptomatology was predominantly gastric with no signs of intestinal occlusion. Due to gravity and large tumour size, both the transverse colon and stomach were pulled by the tumour in the left flank and iliac 


\section{CLINICAL ASPECTS}

fossa. The newly created situation has led to the difficulty of differential diagnosis both from a clinical, paraclinical, ultrasound and tomographic point of view.

The surgical treatment in transverse colon cancer is quite controversial, considering that its vascularization is made from the right colic artery, as well as from the left colic artery. It varies from transverse colectomy, extended right hemicolectomy or left hemicolectomy depending on the location of the tumour and the intention to radicalize the procedure.

When the transverse colon tumour invades the locoregional area, the surgical treatment adapts to the situation, which may also be radical or palliative. $(8,9,10)$ In our case, gastric invasion of the transverse colon tumour has led to great difficulties in differential diagnosis, mimicking a gastric intraparietal tumour, but which does not invade the mucous of the stomach or an extrinsic compression. Segmental colectomy transverse colon and partial gastractomy - resection of the large gastric curvature was performed.

\section{CONCLUSIONS}

Transverse colon cancer can frequently cause locoregional invasion, most frequently affecting the stomach. The predominant symptomatology of gastric pathology, as well as unclear paraclinical data, can lead to difficulties in differential diagnosis related to the starting point of the neoplasia: gastric or colonic.

Surgical treatment is extremely important in terms of radicality, but it depends on the origin of the tumours.

Large colon tumours are generally asymptomatic, therefore they can evolve for a long time without being perceived by the patient until the onset of symptoms related to the invasion of the surrounding organs.

\section{REFERENCES}

1. Siegel RL, Miller KD, Jemal A. Cancer statistics, 2020. CA Cancer J Clin. 2020;70:7

2. Global Burden of Disease Cancer Collaboration, Fitzmaurice C, Allen C, et al. Global, Regional, and National Cancer Incidence, Mortality, Years of Life Lost, Years Lived With Disability, and Disability-Adjusted Lifeyears for 32 Cancer Groups, 1990 to 2015: A Systematic Analysis for the Global Burden of Disease Study. JAMA Oncol. 2017;3:524.

3. Doubeni CA, Laiyemo AO, Major JM, et al. Socioeconomic status and the risk of colorectal cancer: an analysis of more than a half million adults in the National Institutes of Health-AARP Diet and Health Study. Cancer. 2012; $118: 3636$

4. Klabunde $\mathrm{CN}$, Cronin KA, Breen N, et al. Trends in colorectal cancer test use among vulnerable populations in the United States. Cancer Epidemiol Biomarkers Prev. 2011;20:1611.

5. Siegel RL, Miller KD, Jemal A. Colorectal Cancer Mortality Rates in Adults Aged 20 to 54 Years in the United States, 1970-2014. JAMA. 2017;318:572.

6. Ries L, Kosary CL, Hankey BF, et al. SEER cancer statistics review 1973-1995. National Cancer Institute, Bethesda; 1998

7. Mehmet Ali Koc, Suleyman Utku Celik and Cihangir Akyol (September 25th 2019). Colon Cancer, Current Trends in Cancer Management, Liliana Streba, Dan Ionut Gheonea and Michael Schenker, IntechOpen, DOI: 10.5772/intechopen.81597. Available from: https://www.intechopen.com/books/current-trends-incancer-management/colon-cancer.

8. Pin-Chao Wang, Chia-Chi Wang, Ching-Sheng Hsu Colon cancer with gastric invasion mimicking gastric submucosal tumor - GIE, July. 2012;76(1):190-192.

9. Darakhsham A, Lin BP, Chan C, Chapuis PH, Dent OF, Bokey L. Correlates and outcomes of tumor adherence in resected colonic and rectal cancers. Ann Surg. 2008;247:650-8.

10. Michelassi F, Vanmucci L, Ayala J, Chappel R, Goldberg, $\mathrm{R}$, Blocks E. Local recurrence after curative resection of colorectal adenocarcinoma. Surgery. 1990;108:787. 